\title{
Myosin light chain
}

\section{A) Myosin light chain, Original sequence (accession n. X12972):}

1141 acatacaca $\mathbf{C}$ Gggagcaac tcttctgcac aataatttg gacaggtaaa ggttagaaaa

1201 gtgtgtacca catgctatac accatagtag gggctggaat gaccaaaggg tcGttcttga

1261 ataactt $\mathbf{C G}$ acctgggtgg cccaggtcCC ctgatta [aga gGaggggtg gtaggatctg

1321 tgttgaggca cctttcaag tagggagggt ccctggctgt gtgcatggtg ggaggtctct

1381 ggacatttgt atgcattgtc tggtgtㅌct gttcagggcc tgtcagtgcc ccagctgggt

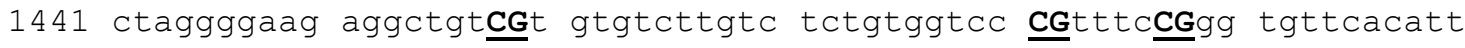

1501 catgtatgtc tgtgtgggtc tgagtgagtg tctgtatatt tatgtctggg tgtttgtatg

1561 cactagtgta tgagtgtctg gatacagcag gcagctgcag tccactcaca gggtcccagg

1621 gtctccagga acaccccaag cacaCGtggc actccctCGa ggactgtcct ccagactagt

1681 tcaacaggct cctttacaa aactcctaag actagtgtct caggttttac acacccacac

1741 t $\underline{\mathbf{C G}} \mathrm{caca \mathbf {CG } \text { GCG}}$ cacacac acacacatgt gtgcacacac acatacatac acactggatt

B) Bisulfite treated sequence with primer annealing sites, bisulfite primer binding sites marked yellow, single nucleotide extension primer binding sites marked red:

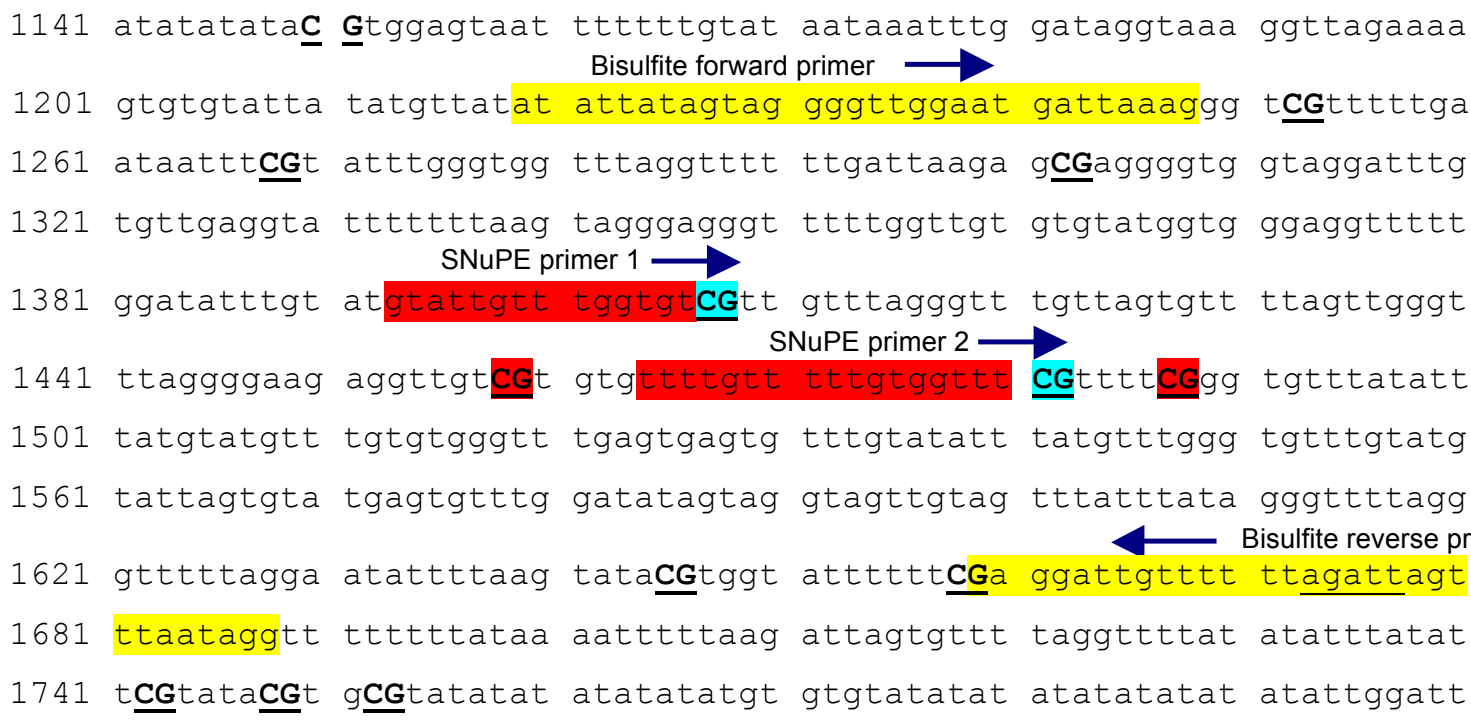




\section{Alpha actin}

\section{A) Alpha actin, Original sequence (accession n. M12347:)}

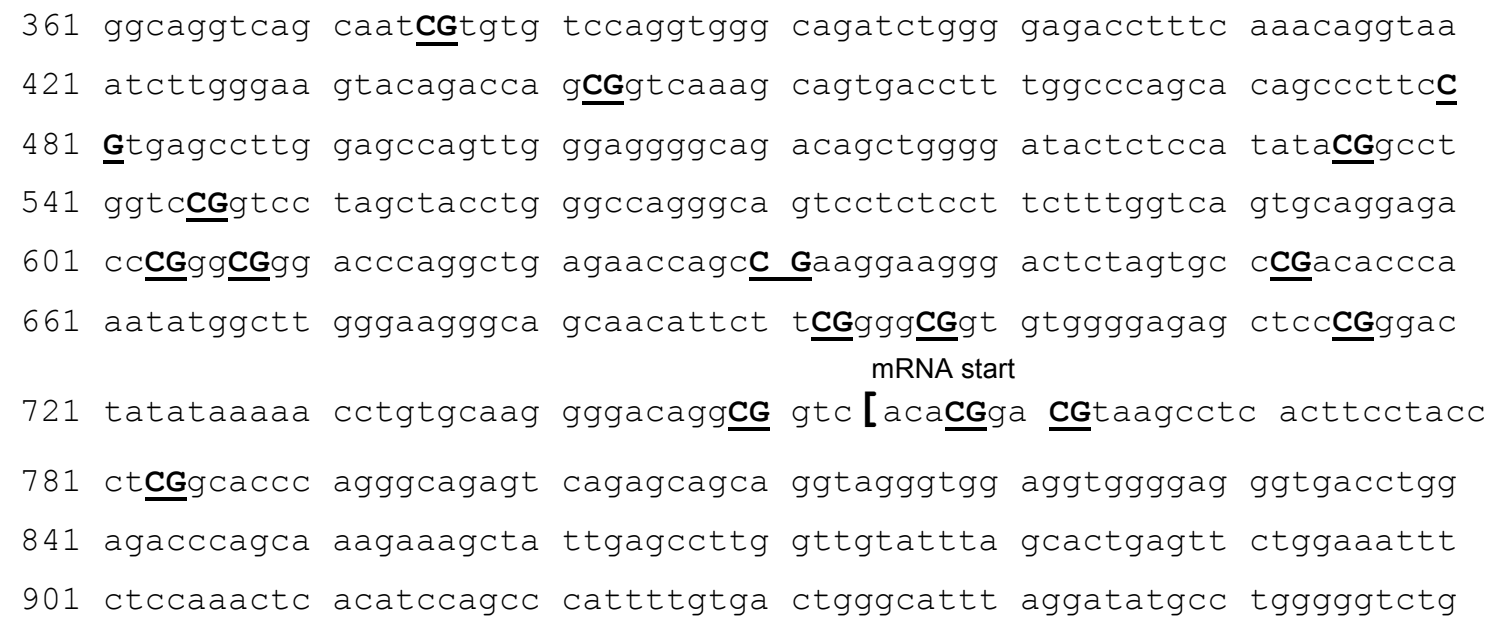

B) Bisulfite treated sequence with primer annealing sites, bisulfite primer binding sites marked yellow, single nucleotide extension primer binding sites marked red:

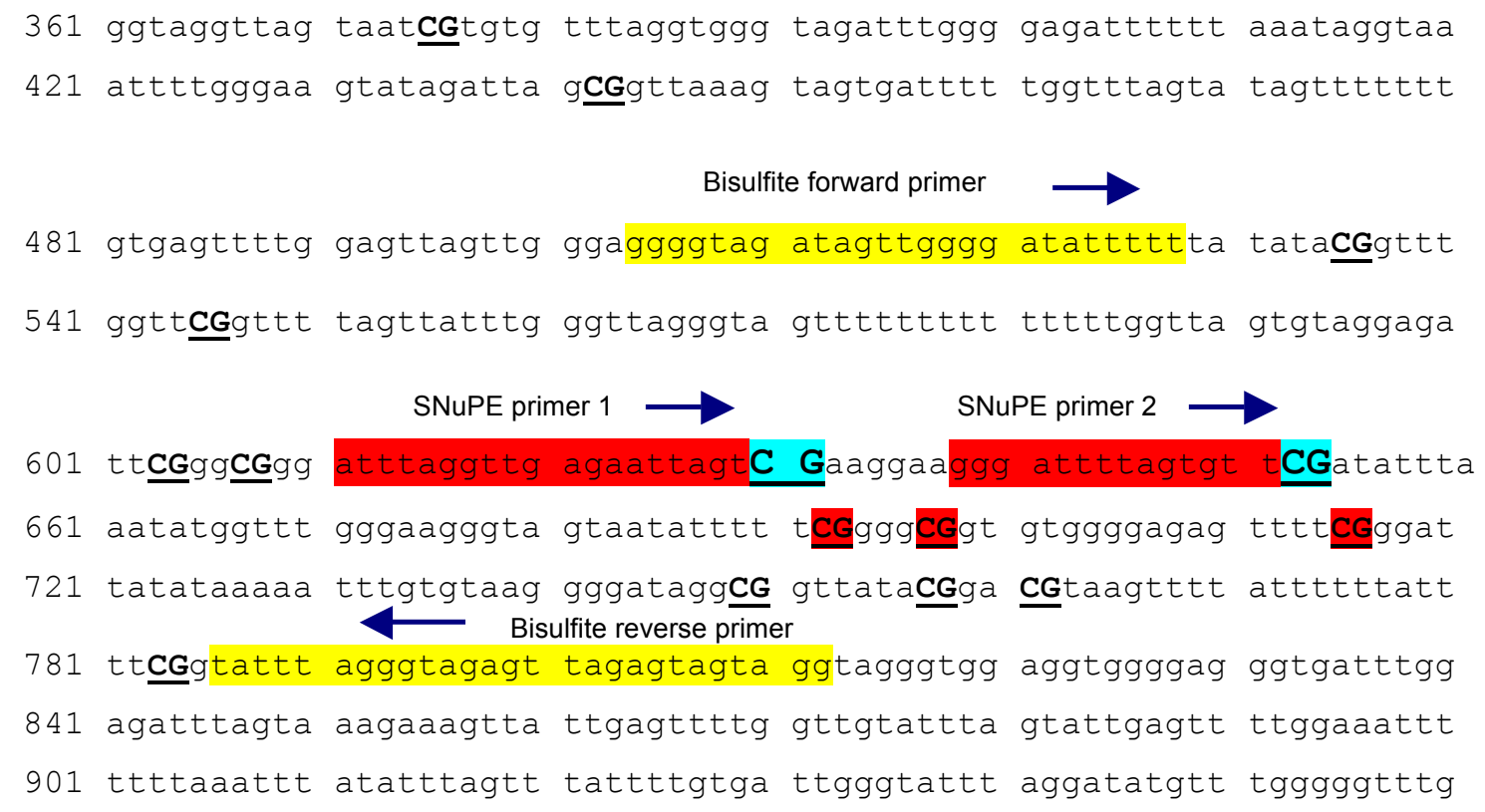


Peg3

A) Peg3, Original Sequence (Accession n. AF105262)

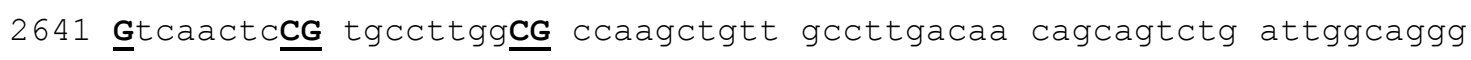

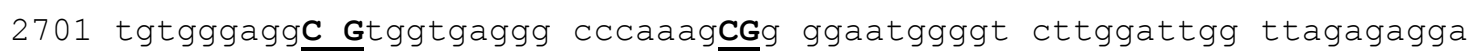
mRNA start

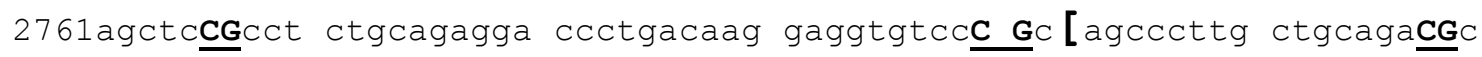

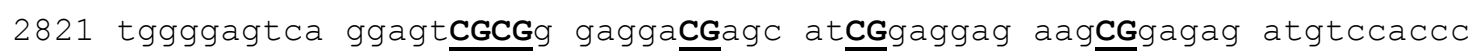

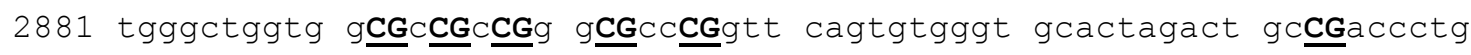

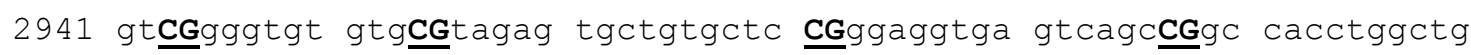

3001 ctctgcagca tgcaccetct tagatac $\underline{\mathbf{C G}}$ ctgcagagtt cagatggtgt ttggggtg $\underline{\mathbf{C G}}$

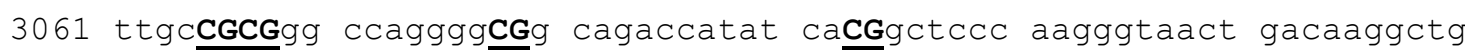

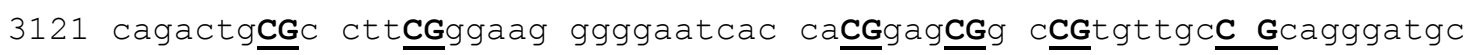

3181 catttaggtg acagggattt aaagtggtat tctataggtc caggcctGg agcctcaggg

B) Bisulfite treated sequence with primer annealing sites, bisulfite primer binding sites marked yellow, single nucleotide extension primer binding sites marked red:

\footnotetext{
Bisulfite forward primer

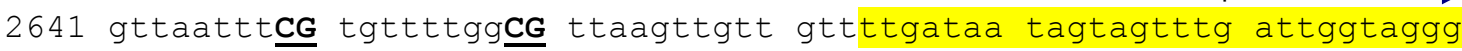

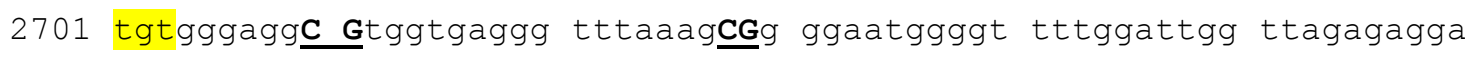

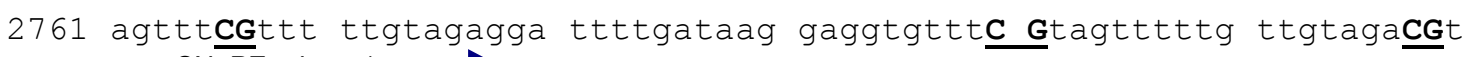
SN $\overline{\text { PPE }}$ primer 1

2821 tggggagtta ggagtCGCGg gaggaCGagt atCGgaggag aagCGgagag atgtttattt SNUPE primer 2

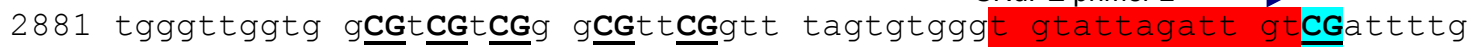

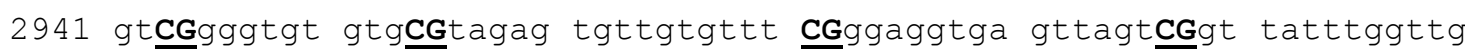

3001 ttttgtagta tgtattttt tagatat $\underline{\mathbf{C G}}$ ttgtagagtt tagatggtgt ttggggtg $\underline{\mathbf{C G}}$

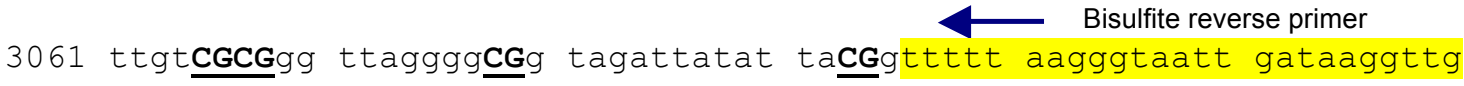

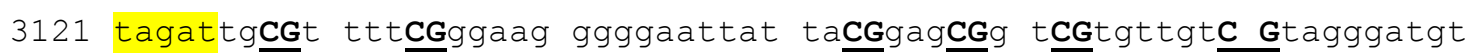




\section{Snrpn}

A) Snrpn, Original Sequence (accession n.AF332579)

67681 aaaalctgt gtgatgcttg caatcacttg ggagcaattt ttttaaaaa ttaaatgtat

67741 ttagtaatag gcaattatat ccattattcc agattgacag tgatttttt tttttaatac

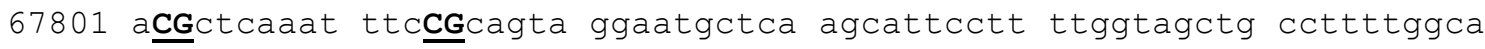

67861 ggacattc $\underline{\mathbf{C G}}$ gtcagaggga cagagacccC tgcattg트

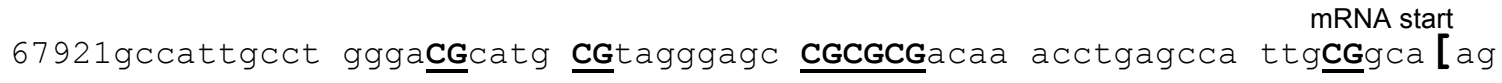

67981 actag $\underline{\mathbf{C G}}$ cag agaggagagg gagC $\underline{\mathbf{C G}}$ gaga tgccaga $\underline{\mathbf{C G}}$ c ttggttctga ggagtgattt

68041 gcaaCGcaat ggag $\underline{\mathbf{C G}}$ agga aggtcagctg ggcttgtgga ttctagtagt gaagtgcat

B) Bisulfite treated sequence with primer annealing sites, bisulfite primer binding sites marked yellow, single nucleotide extension primer binding sites marked red:

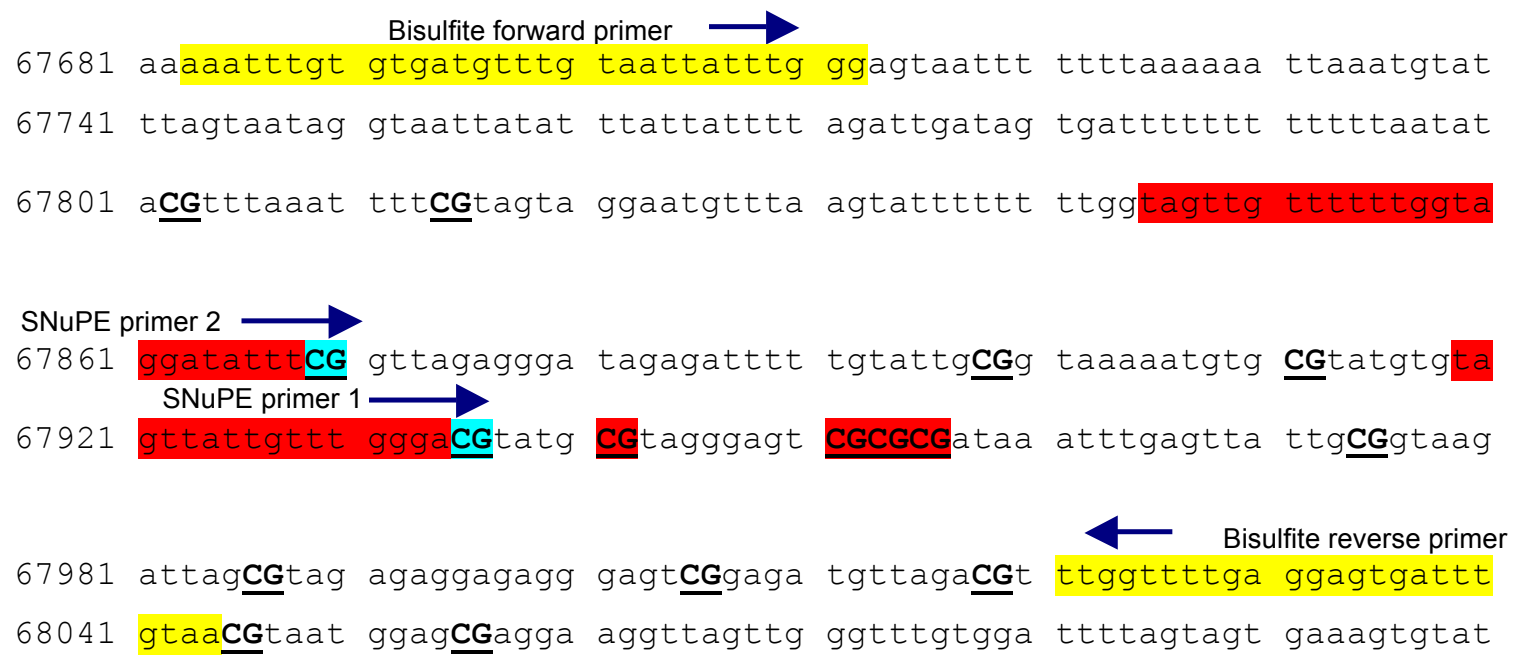


Lit1

A) Lit1, Original Sequence (accession n.AJ271885)

45901 gaacattcㅌ aa $\underline{\mathbf{C G}}$ gagccc ctcactctca gcattaaac agctaccaca taacaacaCG

45961 tactccactc actaccttgg tgctggccac ac $\mathbf{C G g} g c t a c$ aagetcagg ggtctccaga

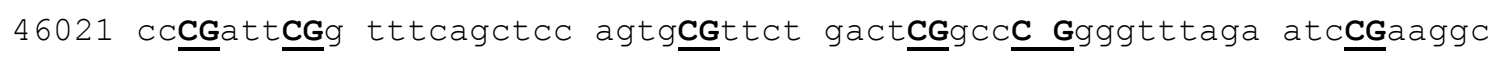

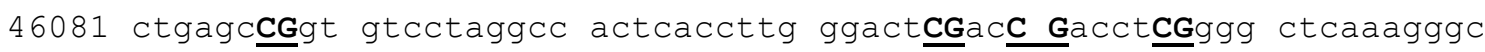

46141 ctcaagacca cccctgcttc tgtaagcctg ggccacaaag atgggga $\underline{\mathbf{C G}}$ ggaCGcaaa

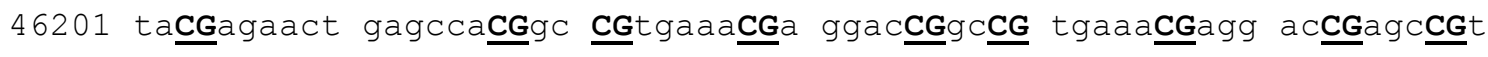

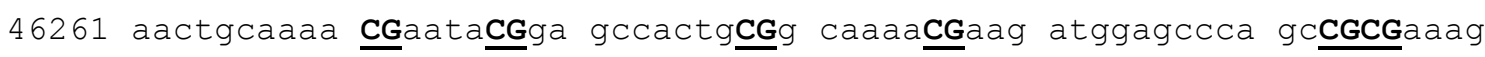

46321 CGCGgcaCGa atcacctctg cttctggC $\underline{\mathbf{C G}}$ tgagtgcttg CGCGaggag ggggaggcta

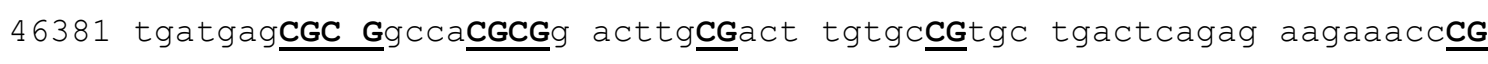

46441 CGctgagaaa aaaaccatac ctaggagaac catgc $\underline{\mathbf{C G}}$ Caga aaagaag $\underline{\mathbf{C G}}$ Ctgggaacca

46501 agctgaacag aaagctctc caagtagaat cacacagagg gaaagaag $\mathbf{C}$ Gtgttgaaga

B) Bisulfite treated sequence with primer annealing sites, bisulfite primer binding sites marked yellow, single nucleotide extension primer binding sites marked red:

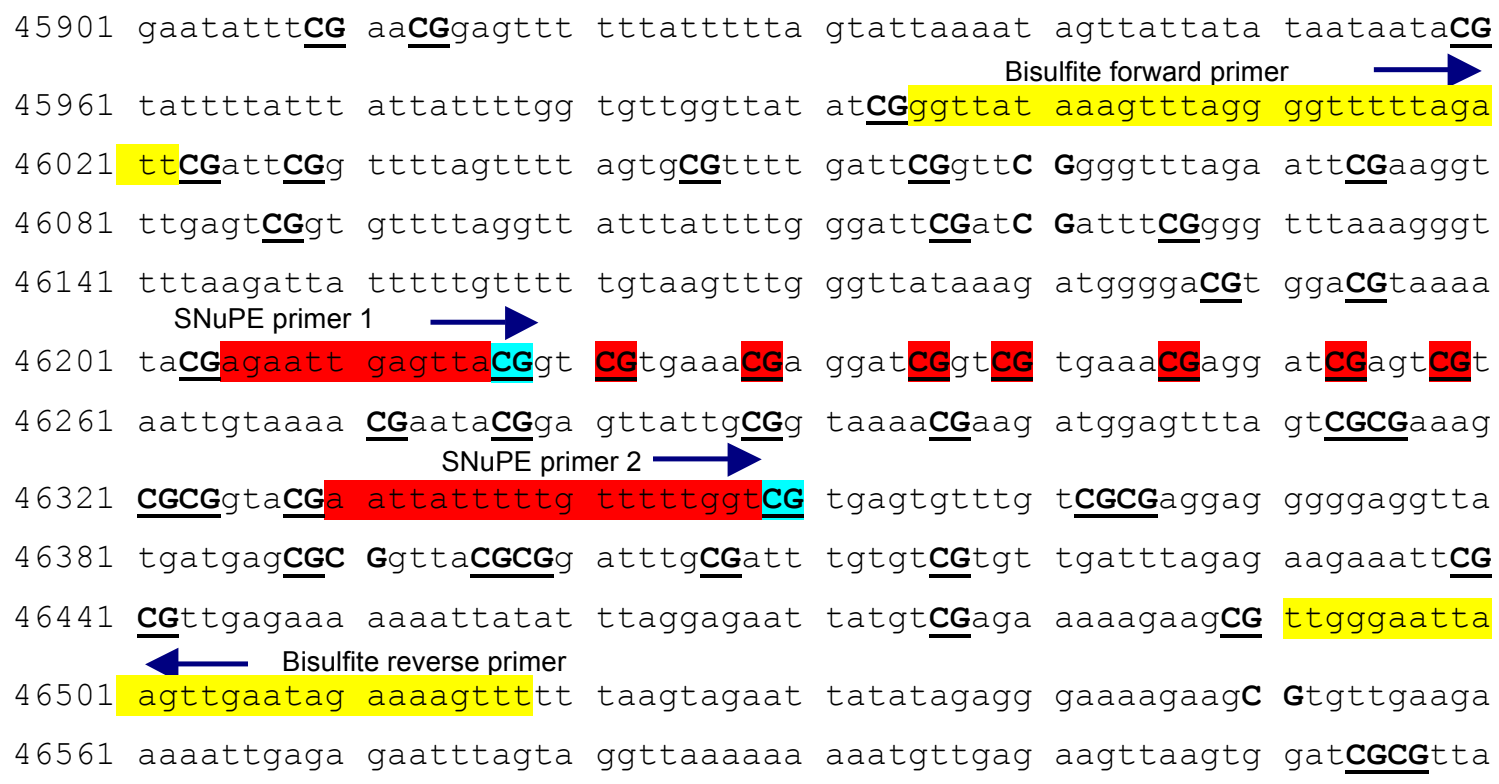




\title{
Line-1
}

\begin{abstract}
A) Line-1 Original Sequence (accession n. D84391)

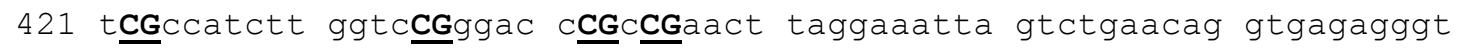
481 g CGccagaga acctgacagc ctctggaaca ggcagaagca cagaggggct gaggcagcac

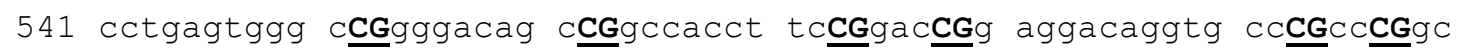

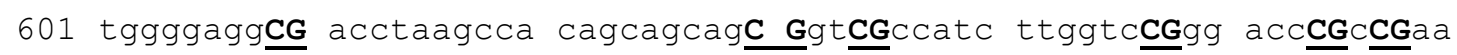
661 cttaggaat tagtctgaac aggtgagagg gtgCGccaga gaacctgaca gcttctggaa

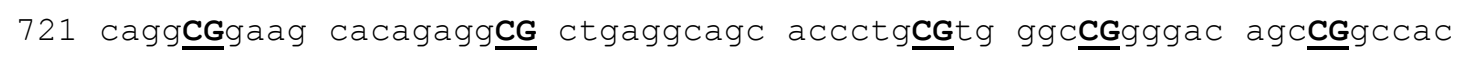

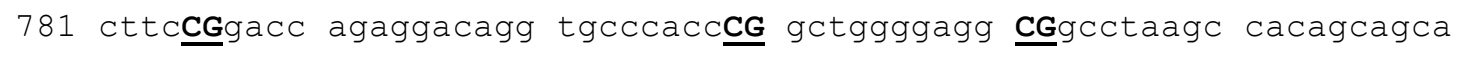
841 g $\underline{\mathbf{C G}} \mathbf{t} \underline{\mathbf{C G}} \mathrm{Cca}$ tcttggtc $\underline{\mathbf{C G}}$ ggacc $\underline{\mathbf{C G}} \underline{\mathbf{C G}}$ aacttaggaa attagtctga acaggtgaga

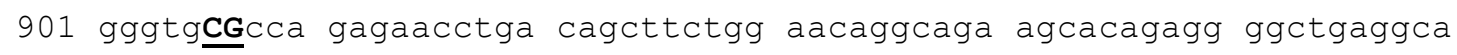

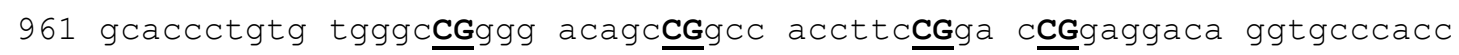

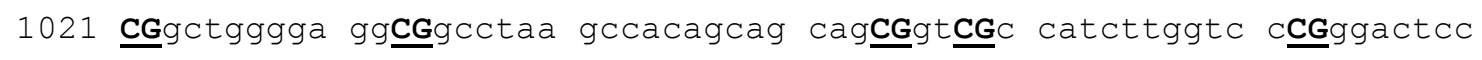
1081 aaggaactta ggaatttagt ctgcttaagt gagagtctgt accacctggg aactgccaaa

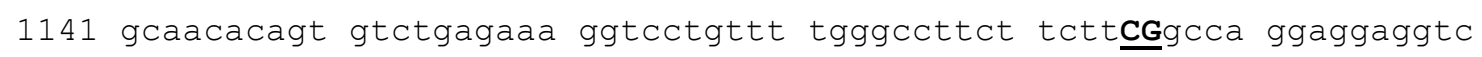

\section{B) Bisulfite treated sequence with primer annealing sites, bisulfite primer binding sites marked yellow, single nucleotide extension primer binding sites marked red:}

421 t $\underline{\mathbf{C G}}$ ttattt ggtt $\underline{\mathbf{C G}} 9 g a t$ t $\underline{\mathbf{C G}} \mathrm{CG}$ aatt taggaaatta gtttgaatag gtgagagggt

Bisulfite forward primer

481 gCGttagaga atttgatagt ttttggaata ggtagaagta tagaggggtt gaggtagtat

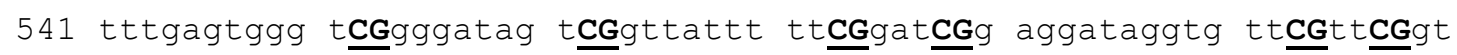

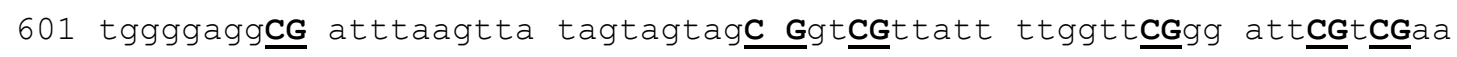

661 tttaggaaat tagtttgaat aggtgagagg gtgCGttaga gaatttgata gtttttggaa

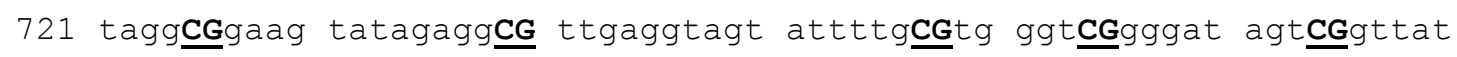

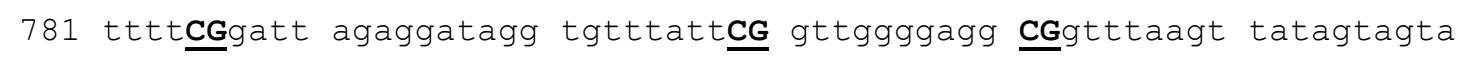

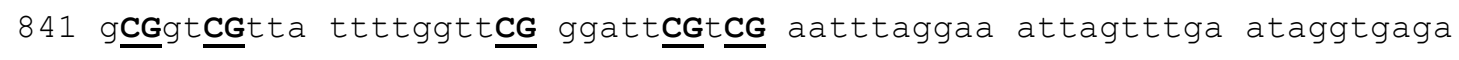

901 gggtg CGtta gagaatttga tagtttttgg aataggtaga agtatagagg ggttgaggta

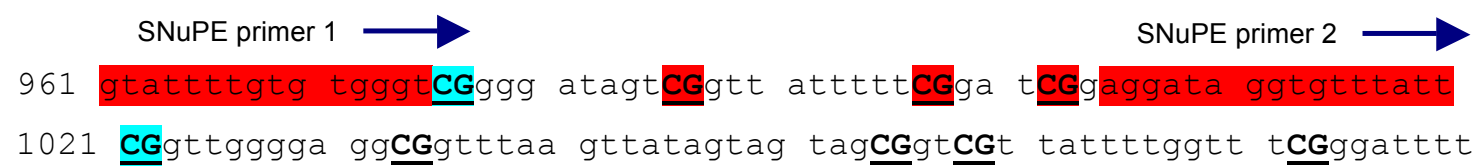

1081 aaggaattta ggaatttagt ttgtttaagt gagagtttgt attatttggg aattgttaaa

1141 gtaatatagt gtttgagaaa ggttttgttt tgggttttt ttt $\underline{\mathbf{C G}}$ tta ggaggaggtt 


\section{IAP (LTR)}

\section{A) Long Tandem repeat of IAP, Original Sequence (accession n. M17551)}

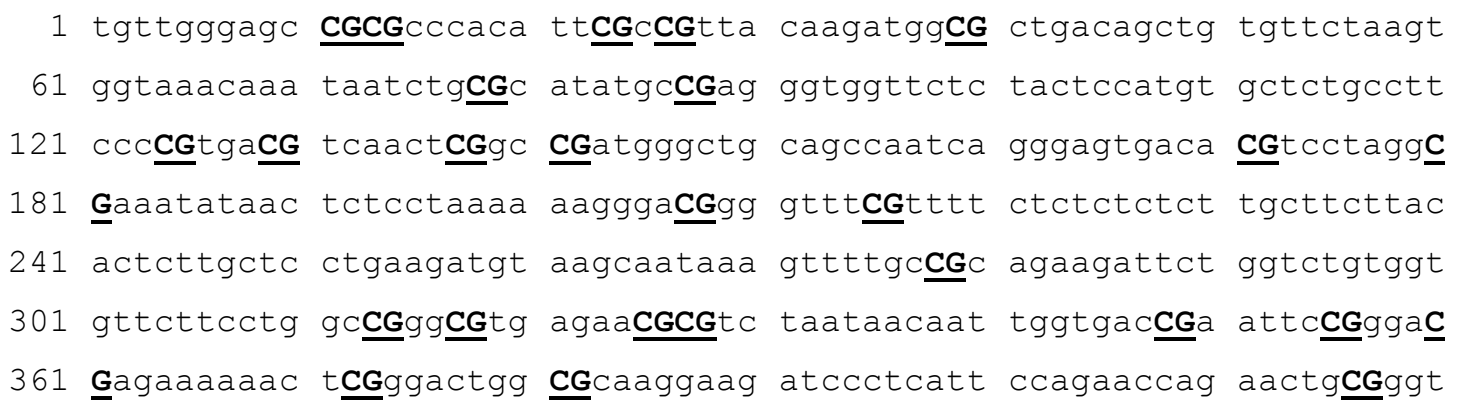

B) Bisulfite treated sequence with primer annealing sites, bisulfite primer binding sites marked yellow, single nucleotide extension primer binding sites marked red:

Bisulfite forward primer

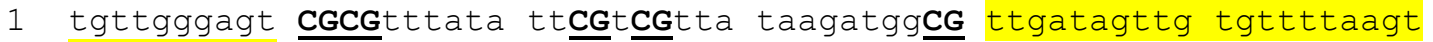

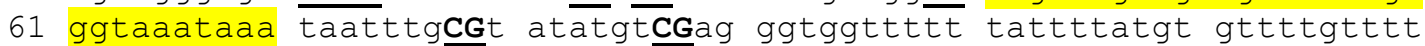

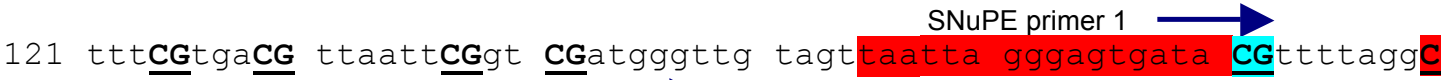

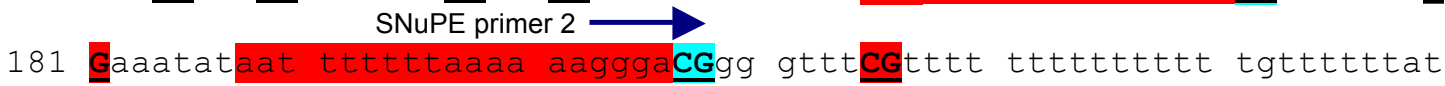

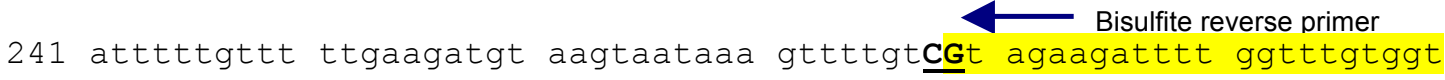

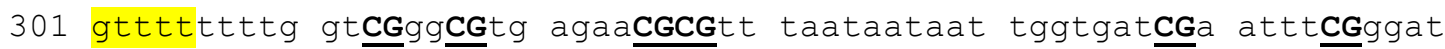

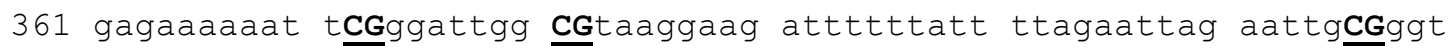

